\title{
MLPA for confirmation of array CGH results and determination of inheritance
}

\author{
Alison Hills ${ }^{1 *}$, Joo Wook Ahn², Celia Donaghue ${ }^{1}$, Helen Thomas ${ }^{1}$, Kathy Mann ${ }^{1}$, Caroline Mackie Ogilvie ${ }^{2}$
}

\begin{abstract}
Background: Array CGH has recently been introduced into our laboratory in place of karyotype analysis for patients with suspected genomic imbalance. Results require confirmation to check sample identity, and analysis of parental samples to determine inheritance and thus assess the clinical significance of the abnormality. Here we describe an MLPA-based strategy for the follow-up of abnormal aCGH results.

Results: In the first 17 months of our MLPA-based aCGH follow-up service, 317 different custom MLPA probes for novel aCGH-detected abnormalities were developed for inheritance studies in 164 families. In addition, 110 samples were tested for confirmation of aCGH-detected abnormalities in common syndromic or subtelomeric regions using commercial MLPA kits. Overall, a total of 1215 samples have been tested by MLPA. A total of 72 de novo abnormalities were confirmed.

Conclusions: Confirmation of aCGH-detected abnormalities and inheritance of these abnormalities are essential for accurate diagnosis and interpretation of aCGH results. The development of a new service utilising custom made MLPA probes and commercial MLPA kits for follow-up studies of array CGH results has been found to be efficient and flexible in our laboratory.
\end{abstract}

\section{Background}

Array Comparative Genomic Hybridisation (aCGH) using either Bacterial Artificial Chromosome (BAC) or oligonucleotide platforms is currently the method of choice for genome-wide screening for chromosome imbalance [1-6]. This technique is now widely used in cytogenetic laboratories as a follow-up test for patients with phenotype suggestive of chromosome imbalance, but with normal karyotypes. An international consensus statement has recently recommended array CGH as a first-line test in place of traditional karyotype analysis [7]; implementation of this recommendation requires that any aCGH diagnostic service should be robust, cost-effective and medium-throughput.

An essential component of such a service is a strategy for confirming abnormal findings, and for establishing the carrier status of the parents. Various strategies for confirmation of abnormal aCGH findings have been described, including repeat aCGH testing [8], Fluorescence In Situ Hybridization (FISH), microsatellite

\footnotetext{
* Correspondence: alison.hills@gsts.com

${ }^{1}$ Cytogenetics Department, GSTS Pathology, London SE1 9RT, UK

Full list of author information is available at the end of the article
}

analysis $[9,10]$, real-time PCR $[11,12]$ and Multiplex Ligation-dependent Probe Amplification (MLPA) for specific genes [8]. Some centres confirm abnormal findings only for small imbalances, due to the confidence associated with abnormal "calls" on multiple adjacent probes (personal communication), whereas others confirm all abnormal findings [1,2].

Inheritance information is essential for accurate interpretation of aCGH findings, and the above strategies can also be applied for testing parental DNA [12-14].

In our laboratory, we have recently introduced oligonucleotide aCGH in place of karyotype analysis [15]. In this paper we describe an MLPA-based approach for inheritance studies, and for confirmation of aCGH abnormalities within common syndromic regions (e.g. chromosome 22q11 microdeletion region) and subtelomeric regions. Our strategy utilises both custom-designed MLPA probes and commercially available MLPA kits used in our existing diagnostic service for products of conception [16] This approach results in considerable time and efficiency savings for our aCGH service. 


\section{Methods}

\section{Array Comparative Genomic Hybridisation}

Array CGH analysis was carried out as described previously [15]. Briefly, DNA was labelled using CGH Labelling Kit for Oligo Arrays (Enzo Life Sciences, USA), then applied to $44 \mathrm{k}$ oligonucleotide arrays (Agilent, USA). Image quantification, array quality control and aberration detection were performed using Feature Extraction and DNA Analytics software packages (Agilent, USA) according to the manufacturer's instructions.

\section{DNA preparation}

All DNA samples for MLPA testing were prepared and quality tested as described previously $[17,18]$. DNA was quantified by spectrophotometry (Nanodrop, USA) and checked for degradation on an agarose gel. Degraded DNA was not used; new samples were requested for these cases.

\section{MLPA custom probe design and synthesis}

Custom MLPA probes were designed online using the publicly available Human MLPA Probe Design algorithm (H-MAPD; http://genomics01.arcan.stonybrook. edu/mlpa/cgi-bin/mlpa.cgi) [19]. Probes of various sizes were designed by specifying the required length of ligation product, with no stuffer sequence. The suggested probe sequences were subjected to BLAT searches http://genome.ucsc.edu/ to verify genomic position and sequence homology. Right hybridizing sequences (RHS) were ordered as 5' phosphorylated "Ultramer" oligonucleotides and left hybridizing sequences (LHS) were ordered as standard desalted oligonucleotides (Integrated DNA Technologies, Belgium).

\section{Custom MLPA assay selection}

MRC-Holland manufacture two MLPA kits, P200 and P300, designed for use with custom MLPA probes. These kits combine reference probes for data normalization and quantity/quality control fragments with space for the addition of multiple custom probes. Both kits were trialled and a final decision made to routinely use the P200 kit as it allowed shorter and therefore cheaper custom probes to be used than the P300 kit. The P300 kit is preferred by users who observe significant effects of signal sloping on their data and counters this problem by distributing reference probes over the whole size range of the probemix. This signal sloping effect was not observed to a significant degree with our data.

Up to three custom MLPA probes, differing in size by at least ten base pairs, were run concurrently in a single probe mix (see Figure 1). This reduced costs by reducing the number of normal control reactions and the associated consumables needed. The potential for making efficiency and consumable savings by increasing the number of probes per mix must be weighted against the cost of probes of increasing size. In our laboratory the most efficient scenario involves the use of three separate four probe mixes, with three family members tested per probe. This allows 12 families to be tested per 96 well plate.

\section{Multiplex Ligation-dependent Probe Amplification}

MLPA reactions using MRC-Holland SALSA ${ }^{\oplus}$ P245, P36E and P69 kits were carried out according to the manufacturer's instructions and as described previously [17].

Custom MLPA probes were prepared and diluted and reactions performed according to the synthetic MLPA protocol provided on the MRC-Holland website http:// mlpa.com.

No optimisation of custom probe concentration was required. Peak heights were found to be generally comparable to those of the P200 reference probes when probes were added at the concentrations recommended by MRC-Holland.

DNA samples tested with custom probes were tested in duplicate; two normal control samples, each tested in triplicate, and a negative control were included for every custom probe mix. The proband was always tested alongside parental samples to confirm assay sensitivity.

\section{MLPA data analysis}

A $3 \mu \mathrm{l}$ aliquot of PCR product was mixed with $0.3 \mu \mathrm{l} \mathrm{Gen-}$ escan 500 LIZ size standard (Applied Biosystems, UK) and $15 \mu \mathrm{l} \mathrm{HiDi}$ formamide (Applied Biosystems, UK) before being size-separated by capillary electrophoresis on a 3730 genetic analyser (Applied Biosystems, UK) according to the manufacturer's instructions.

MLPA peak height data was analysed using GeneMarker v1.8 software (SoftGenetics, USA). A maximum raw data peak height of 15,000 relative fluorescent units ( $\mathrm{rfu}$ ) was established after observing a loss of peak height relative dosage with increasing concentrations of DNA. Peak heights which were too low also produced unreliable dosage ratios; a lower peak height limit was set at $250 \mathrm{rfu}$. Results with peak heights outside of these limits were failed and the sample re-processed.

\section{P36, P69 and P245 MLPA kits}

Data from the P36E and P69 subtelomere kits and the P245 microdeletion/microduplication kit were interpreted according to analysis parameters described previously [18].

\section{Custom MLPA}

Peak height data from MRC-Holland P200 kits with added custom probes were analysed according to recommendations on data normalization provided on the MRC-Holland website. Panels were designed to detect one or more custom probes per kit. 
a)
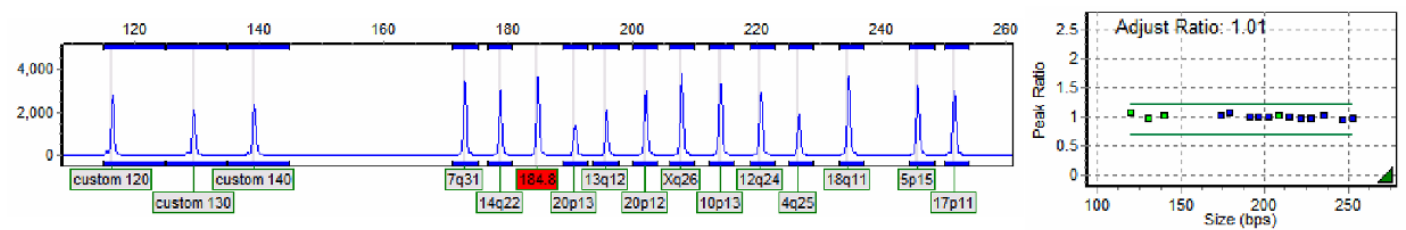

b)
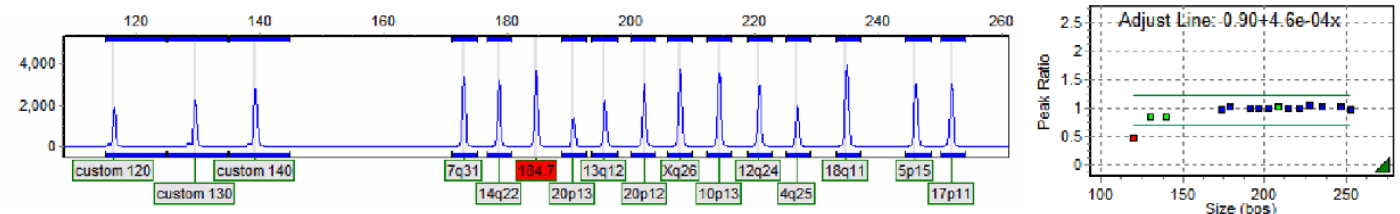

C)
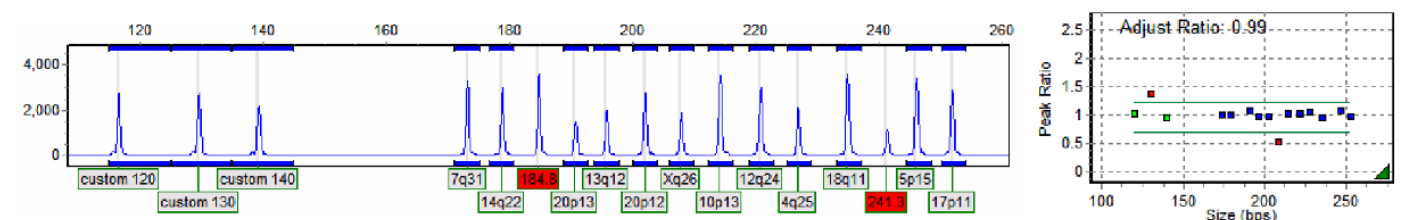

d)
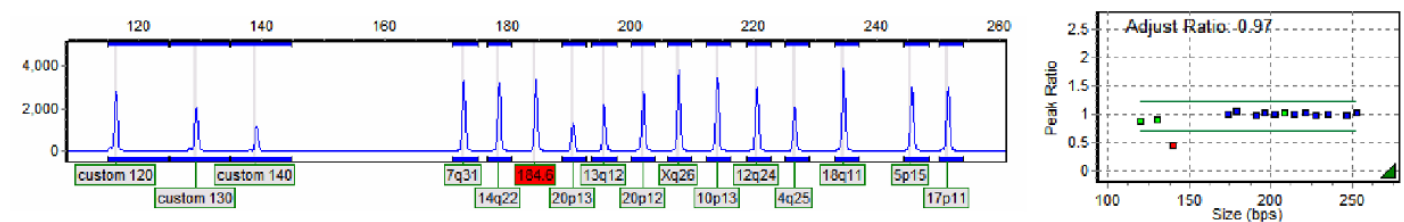

e)
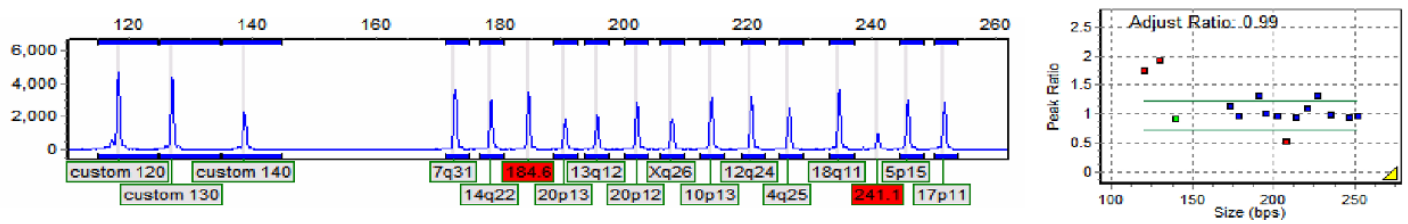

Figure 1 MLPA results of three custom probes in the MRC-Holland P200 kit. GeneMarker electrophoretograms are shown on the left, normalized MLPA data on the right. The $184 \mathrm{bp}$ peak represents the D-fragment which is used to highlight incomplete denaturation and is not shown in the analysed data on the right, the $241 \mathrm{bp}$ fragment is a Y chromosome specific fragment for the identification of male samples. a) normal female control sample b) female sample with decreased copy number for the $120 \mathrm{bp}$ probe c) male sample with increased copy number for the $130 \mathrm{bp}$ probe d) female sample with decreased copy number for the 140 bp probe e) poor quality male sample with several outlying probes.

The internal control normalization function was selected so that sample data was only compared to userspecified control data rather than to the whole run. Custom probe peaks were not included in the normalization

The MLPA Analysis function in GeneMarker was used for determining copy number of custom probes with MLPA peak height ratios defined as deletion $<0.71 \leq$ normal $\leq 1.23 \leq$ duplication (as described by [17]). Figure 1 shows an example trace.

For a result to be called, at least one out of three replicates for each control sample must pass (i.e. peak height within limits and no outlying probe peaks), and both duplicate patient results must be concordant. Samples with reference probe peak ratios which fell outside of the normal range were failed (see Figure 1e), as were samples with high QC fragment peaks.

This analysis approach is similar to that used for our diagnostic service for the molecular analysis of miscarriage products [16].

\section{Genotyping}

Genotyping for relationship testing (in families where the abnormality in the proband had apparently arisen de novo) was carried out using multiplex quantitative fluorescence PCR (QF-PCR) assays containing multiple polymorphic STR markers which are routinely used in our department for aneuploidy diagnosis [20,21].

\section{Results}

In the period from January 2009 to May 2010, 3306 array CGH tests were carried out in our laboratory. The following sections refer to MLPA results obtained within this time period.

\section{Confirmation of array CGH results and inheritance studies using custom MLPA probes}

When parental blood samples were received for followup studies of aCGH findings not covered by probes in MRC-Holland P36, P69 or P245 kits, a custom MLPA 
probe specific for a locus within the abnormal region was designed. Potentially relevant genes were targeted and common copy number variants $(\mathrm{CNV})$ were avoided.

During this period, 317 unique custom MLPA probes were designed for 164 families in order to determine inheritance of both duplication and deletion results, and were tested on a total of 1105 individuals. Figure 2 depicts the spread of custom probes across the genome.

The majority of custom probes were used for only single families; however, certain regions have started to emerge as exhibiting recurrent deletions and/or duplications. This may be due to previously unidentified recurrent syndromic regions, or may represent benign population polymorphisms. To date, the probe with the greatest usage is located at Xp22.31. This has been used for 11 families. Duplication of this region has been found in patients with a range of different phenotypes, and also in normal parents, both male and female, and has therefore been suggested as a benign variant [22]. The reciprocal deletion gives rise to steroid sulphatase deficiency in male carriers (OMIM 306480).
In addition, a custom probe has been designed for the autism susceptibility locus on chromosome 16p11.2 [23-25]. Copy number changes within this region are frequently detected by aCGH and are very likely to arise following unequal meiotic crossing over between flanking low-copy repeats. Custom MLPA can therefore be used to confirm the abnormality, thus eliminating the need for repeat aCGH testing in such cases. Within our data set, this custom MLPA probe has been used to confirm copy number changes and for inheritance studies in five families.

Twenty seven custom MLPA probes (8.5\%) failed, due to either amplification failure or inconsistent results. In all cases, replacement probes were designed.

\section{Confirmation of subtelomeric abnormalities and common microdeletion/microduplications detected by aCGH}

During this period, 49 samples from 28 families were tested using subtelomere kits (P36E and P69) for followup of aCGH results. Three de novo terminal abnormalities were confirmed out of a total of nine where inheritance studies have been completed; a chromosome 9p deletion,

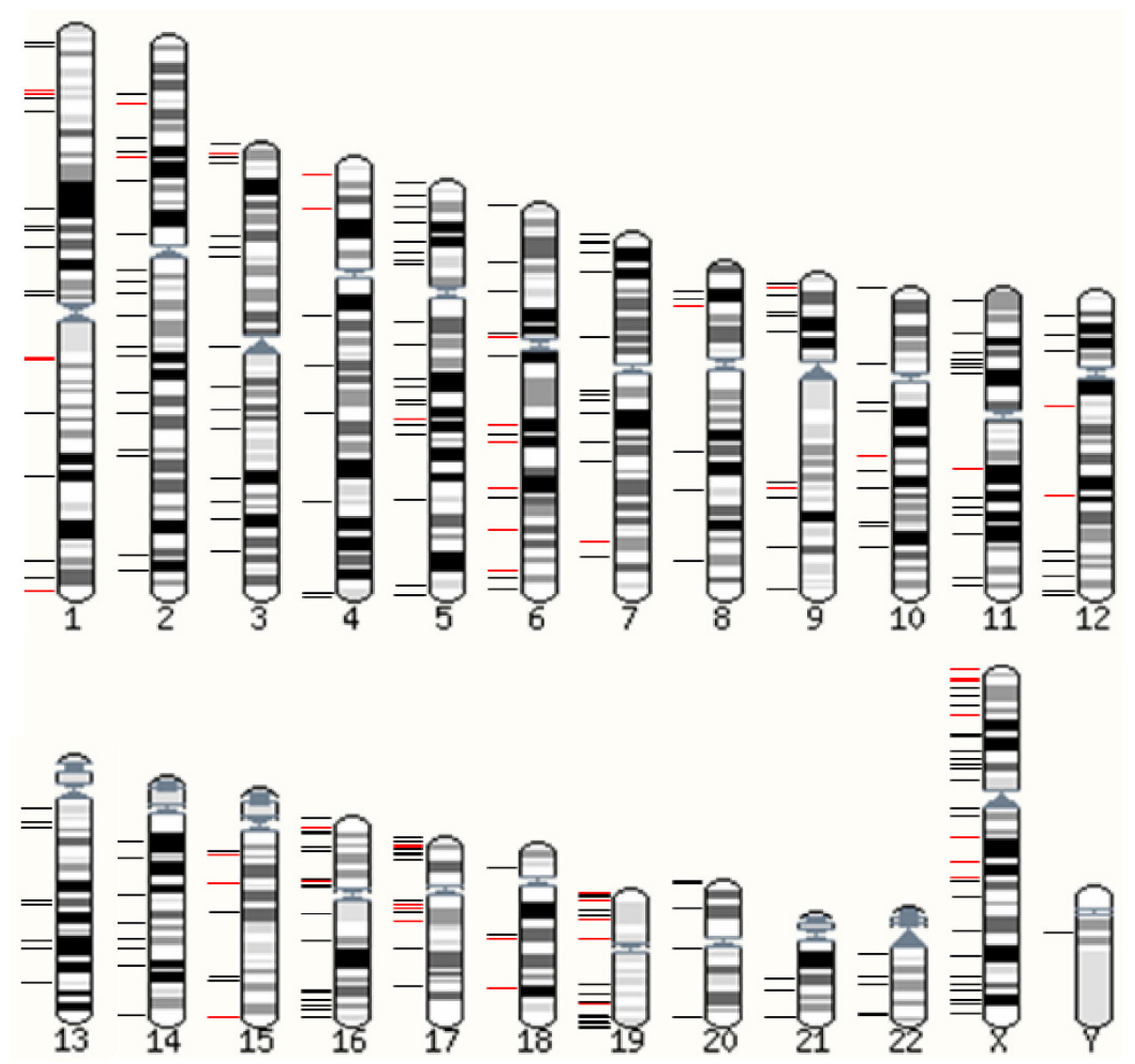

Figure 2 Karyotype illustrating the spread of custom probes across the genome. Black bars indicate that a probe has been tested on one family, red bars indicate that a probe has been tested on more than one family. 
a proximal chromosome 13q deletion (confirmed using the P36E kit which contains a probe for the proximal region of the long arm of chromosome 13) and an unbalanced translocation between the telomeric regions of the short arms of chromosomes 5 and 8 .

In the same period, 61 samples from 32 families were tested using microdeletion/microduplication kits (P245) for follow-up of aCGH results. Eleven de novo results were confirmed out of a total of twelve where inheritance studies had been completed; six deletions of the chromosome 22q11 microdeletion/microduplication region, three abnormalities in the Prader Willi/Angelman syndrome region, one duplication of the SmithMagenis region, one partial deletion of the Miller-Dieker region, one deletion of the NF1 region, one deletion of the Alagille region and one duplication of the Williams Beuren region. One case of an inherited chromosome 22q11 duplication was also confirmed.

\section{Discrepant results}

We observed no discrepancies between array CGH results and MLPA results. Apparent discrepancies may arise due to SNPs close to the ligation site of the two probe hybridising sequences, causing amplification failure, and therefore mimicking a deleted result. This is mitigated by using the H-MAPD software which checks potential probe sequences against the latest SNP database.

\section{Results turnaround times}

Confirmation and inheritance results were generally available within two weeks of sample receipt. Probes were usually synthesized and dispatched by IDT within five working days of ordering. Tests using existing stocks of probes could be turned around in less than a week in urgent cases. The rate of repeat testing due poor DNA quality or discordant probe results was $14 \%$.

\section{Discussion}

Array CGH testing for genomic imbalance has been introduced as a first-line test in place of G-banded chromosome analysis in a number of centres, and is offered as an additional test by most cytogenetics laboratories. As with any test protocol that involves the simultaneous handling of multiple DNA samples, it is important that any abnormal results are confirmed in order to establish patient identity before reporting. However, repeat array CGH testing of all abnormal findings, and array testing of parental samples for inheritance studies adds significantly to the cost of the service and may also result in incidental findings potentially requiring further investigation.

MLPA is now an established technique for the investigation of copy number [26]. MRC-Holland http://www. mrc-holland.com manufactures numerous commercial
MLPA kits for the detection of copy number changes in different genomic locations. We currently use three of these kits (P36E, P69 and P245) in our recently described diagnostic service for the molecular analysis of miscarriage products [16]. Prior to the introduction of aCGH into our laboratory we also used these kits for the diagnosis of subtelomeric imbalance (P36E and P69) and common microdeletion/microduplication syndromes (P245) in patients with idiopathic developmental delay and/or dysmorphism [17]. We therefore have in operation in our laboratory an efficient and medium throughput diagnostic MLPA-based service and have refined our sample preparation and data analysis techniques to improve accuracy and turnaround times. We were able to exploit this existing service in order to develop an efficient follow-up strategy for aCGH testing. However, the approach we describe should also be feasible in centres with an appropriate genetic analyser without previous experience of this technique, as extensive technical support is available from MRC-Holland, and DNA preparation, probe design, reaction set up and data analysis protocols are readily available. In our experience, optimisation is not required for the majority of custom MLPA probes. Furthermore, the use of custom MLPA probes represents a simple method for establishing copy number beyond the usage described in this paper.

This approach results in significant time and cost savings by avoiding the need for aCGH testing for confirmation of common abnormalities or of parental samples. Other follow-up approaches that have been described include FISH, which has the advantage of giving position information, and excluding balanced rearrangements in parents, in cases of apparent de novo findings [13]. However, this approach requires cell culture in order to examine chromosome spreads, whereas MLPA is performed on the same DNA sample as is used for the original aCGH analysis; in addition some regions of imbalance detected by aCGH are too small to be followed up by routinely-available FISH probes. Nevertheless, where FISH probes are available, we offer FISH testing to parents of children with de novo abnormalities, in order to refine their reproductive risk; no families have yet requested this testing. We have compared real-time PCR as a follow-up strategy and have found it to be more costly and time-consuming than MLPA (results not shown).

Follow-up of aCGH results using STR markers and QF-PCR is limited by a lack of polymorphic markers, time taken for optimization and the inability to confirm deletions in the absence of parental genotypes. However, we are able to confirm common trisomies using the multiplex QF-PCR kits designed for our prenatal service $[20,27]$. 


\section{Conclusions}

Follow-up of abnormal aCGH results is good practice to confirm patient identity, and inheritance studies are necessary for accurate interpretation of these abnormal findings. The results presented here demonstrate that MLPA is a fast, robust and accurate technique for confirmation and follow-up of both duplication and deletion aCGH results. However, exclusion of balanced parental rearrangements by FISH is recommended for apparently de novo abnormalities.

\section{Acknowledgements}

We thank members of the Cytogenetics Department for their input.

\section{Author details \\ ${ }^{1}$ Cytogenetics Department, GSTS Pathology, London SE1 9RT, UK. ${ }^{2}$ Cytogenetics Department, Guy's \& St Thomas' NHS Foundation Trust, London SE1 9RT, UK.}

\section{Authors' contributions}

AH developed the custom MLPA probe approach, designed the probes, analysed the data and wrote the initial draft of the paper. JWA developed the MLPA diagnostic analysis strategy and helped with the data analysis for the paper. CD helped to develop the custom MLPA probe approach and design the custom MLPA probes, and carried out the diagnostic interpretation. HT carried out the routine testing and assisted with interpretation. KM initiated MLPA testing in our laboratory, helped with development of the MLPA diagnostic analysis strategy, and advised on the development of this service. CMO initiated and led the development of this service, and co-wrote the paper.

All the authors have provided input for the paper and have read and approved the final manuscript.

\section{Competing interests}

The authors declare that they have no competing interests.

Received: 25 August 2010 Accepted: 13 October 2010

Published: 13 October 2010

\section{References}

1. Baris HN, Tan WH, Kimonis VE, Irons MB: Diagnostic utility of array-based comparative genomic hybridization in a clinical setting. Am J Med Genet A 2007, 143A:2523-2533.

2. Fan YS, Jayakar P, Zhu H, Barbouth D, Sacharow S, Morales A, Carver V, Benke $P$, Mundy $P$, Elsas $L J$ : Detection of pathogenic gene copy number variations in patients with mental retardation by genomewide oligonucleotide array comparative genomic hybridization. Hum Mutat 2007, 28:1124-1132.

3. Jaillard S, Drunat $S$, Bendavid C, Aboura A, Etcheverry A, Journel $H$, Delahaye A, Pasquier L, Bonneau D, Toutain A, et al: Identification of gene copy number variations in patients with mental retardation using arrayCGH: Novel syndromes in a large French series. Eur I Med Genet 2010, 53:66-75.

4. Pickering DL, Eudy JD, Olney AH, Dave BJ, Golden D, Stevens J, Sanger WG: Array-based comparative genomic hybridization analysis of 1176 consecutive clinical genetics investigations. Genet Med 2008, 10:262-266.

5. Shen Y, Irons M, Miller DT, Cheung SW, Lip V, Sheng X, Tomaszewicz K, Shao $H$, Fang $H$, Tang HS, et al: Development of a focused oligonucleotide-array comparative genomic hybridization chip for clinical diagnosis of genomic imbalance. Clin Chem 2007, 53:2051-2059.

6. Miyake N, Shimokawa O, Harada N, Sosonkina N, Okubo A, Kawara H, Okamoto N, Kurosawa K, Kawame H, Iwakoshi M, et al: BAC array CGH reveals genomic aberrations in idiopathic mental retardation. Am J Med Genet A 2006, 140:205-211.

7. Miller DT, Adam MP, Aradhya S, Biesecker LG, Brothman AR, Carter NP, Church DM, Crolla JA, Eichler EE, Epstein CJ, et al: Consensus statement: chromosomal microarray is a first-tier clinical diagnostic test for individuals with developmental disabilities or congenital anomalies. Am J Hum Genet 2010, 86:749-764.

8. Siggberg L, Ala-Mello S, Jaakkola E, Kuusinen E, Schuit R, Kohlhase J, Bohm D, Ignatius J, Knuutila S: Array CGH in molecular diagnosis of mental retardation - A study of 150 Finnish patients. Am J Med Genet A 2010, 152A:1398-1410.

9. Schluth C, Gesny R, Borck G, Redon R, Abadie V, Kleinfinger P, Munnich A, Lyonnet $S$, Colleaux L: New case of interstitial deletion 12(q15-q21.2) in a girl with facial dysmorphism and mental retardation. Am J Med Genet A 2008, 146A:93-96.

10. Sebat J, Lakshmi B, Malhotra D, Troge J, Lese-Martin C, Walsh T, Yamrom B, Yoon S, Krasnitz A, Kendall J, et al: Strong association of de novo copy number mutations with autism. Science 2007, 316:445-449.

11. Yu S, Kielt M, Stegner AL, Kibiryeva N, Bittel DC, Cooley LD: Quantitative real-time polymerase chain reaction for the verification of genomic imbalances detected by microarray-based comparative genomic hybridization. Genet Test Mol Biomarkers 2009, 13:751-760.

12. Thienpont B, Mertens L, de Ravel T, Eyskens B, Boshoff D, Maas N, Fryns JP, Gewillig M, Vermeesch JR, Devriendt K: Submicroscopic chromosomal imbalances detected by array-CGH are a frequent cause of congenital heart defects in selected patients. Eur Heart J 2007, 28:2778-2784.

13. Kang SH, Shaw C, Ou Z, Eng PA, Cooper ML, Pursley AN, Sahoo T, Bacino CA, Chinault AC, Stankiewicz P, et al: Insertional translocation detected using FISH confirmation of array-comparative genomic hybridization (aCGH) results. Am J Med Genet A 2010, 152A:1111-1126.

14. Reddy S, Dolzhanskaya N, Krogh J, Velinov M: A novel $1.4 \mathrm{Mb}$ de novo microdeletion of chromosome 1q21.3 in a child with microcephaly, dysmorphic features and mental retardation. Eur J Med Genet 2009, 52:443-445.

15. Ahn JW, Mann K, Walsh S, Shehab M, Hoang S, Docherty Z, Mohammed S, Mackie Ogilvie $C$ : Validation and implementation of array comparative genomic hybridisation as a first line test in place of postnatal karyotyping for genome imbalance. Mol Cytogenet 2010, 3:9.

16. Donaghue C, Mann K, Docherty Z, Mazzaschi R, Fear C, Ogilvie C: Combined QF-PCR and MLPA molecular analysis of miscarriage products: an efficient and robust alternative to karyotype analysis. Prenat Diagn 2010, 30:133-137.

17. Ahn JW, Ogilvie CM, Welch A, Thomas H, Madula R, Hills A, Donaghue C, Mann K: Detection of subtelomere imbalance using MLPA: validation, development of an analysis protocol, and application in a diagnostic centre. BMC Med Genet 2007, 8:9.

18. Ahn JW, Mann K, Docherty Z, Mackie Ogilvie C: Submicroscopic chromosome imbalance in patients with developmental delay and/or dysmorphism referred specifically for Fragile $\mathrm{X}$ testing and karyotype analysis. Mol Cytogenet 2008, 1:2.

19. Zhi J, Hatchwell E: Human MLPA Probe Design (H-MAPD): a probe design tool for both electrophoresis-based and bead-coupled human multiplex ligation-dependent probe amplification assays. BMC Genomics 2008, 9:407.

20. Mann K, Fox SP, Abbs SJ, Yau SC, Scriven PN, Docherty Z, Ogilvie CM: Development and implementation of a new rapid aneuploidy diagnostic service within the UK National Health Service and implications for the future of prenatal diagnosis. Lancet 2001, 358:1057-1061.

21. Ogilvie CM, Donaghue C, Fox SP, Docherty Z, Mann K: Rapid prenatal diagnosis of aneuploidy using quantitative fluorescence-PCR (QF-PCR). J Histochem Cytochem 2005, 53:285-288.

22. Li F, Shen Y, Kohler U, Sharkey FH, Menon D, Coulleaux L, Malan V, Rio M, McMullan DJ, Cox H, et al: Interstitial microduplication of Xp22.31: Causative of intellectual disability or benign copy number variant? Eur J Med Genet 2010, 53:93-99.

23. McCarthy SE, Makarov V, Kirov G, Addington AM, McClellan J, Yoon S, Perkins DO, Dickel DE, Kusenda M, Krastoshevsky O, et al: Microduplications of 16p11.2 are associated with schizophrenia. Nat Genet 2009, 41:1223-1227.

24. Shinawi M, Liu P, Kang SH, Shen J, Belmont JW, Scott DA, Probst FJ, Craigen WJ, Graham BH, Pursley A, et al: Recurrent reciprocal 16p11.2 rearrangements associated with global developmental delay, behavioural problems, dysmorphism, epilepsy, and abnormal head size. J Med Genet 2010, 47:332-341. 
25. Weiss $L A$, Shen $Y$, Korn $J M$, Arking DE, Miller DT, Fossdal R, Saemundsen E, Stefansson H, Ferreira MA, Green T, et al: Association between microdeletion and microduplication at 16p11.2 and autism. N Engl J Med 2008, 358:667-675.

26. Schouten JP, McElgunn CJ, Waaijer R, Zwijnenburg D, Diepvens F, Pals G: Relative quantification of 40 nucleic acid sequences by multiplex ligation-dependent probe amplification. Nucleic Acids Res 2002, 30:e57.

27. Donaghue C, Roberts A, Mann K, Ogilvie CM: Development and targeted application of a rapid QF-PCR test for sex chromosome imbalance. Prenat Diagn 2003, 23:201-210.

doi:10.1186/1755-8166-3-19

Cite this article as: Hills et al: MLPA for confirmation of array CGH results and determination of inheritance. Molecular Cytogenetics 2010 3:19.

Submit your next manuscript to BioMed Central and take full advantage of:

- Convenient online submission

- Thorough peer review

- No space constraints or color figure charges

- Immediate publication on acceptance

- Inclusion in PubMed, CAS, Scopus and Google Scholar

- Research which is freely available for redistribution

Submit your manuscript at www.biomedcentral.com/submit 